\title{
EFECTO DE LA ALTURA DE DEFOLIACIÓN SOBRE EL RENDIMIENTO ESTACIONAL DE Stylosanthes guianensis cv. Graham.
}

\author{
CIOTTI, Elsa M. ${ }^{1}$; TEPPER, Norma ${ }^{1}$; PORTA, Miriam²; CASTELAN, María E².; HACK, Claudina M. ${ }^{2}$ \\ ${ }^{1}$ Cátedra de Forrajicultura FCA- UNNE. - Sgto Cabral 2131- Corrientes. Argentina. \\ ${ }^{2}$ Instituto Agrotécnico "Pedro M. Fuentes Godo" FCA-UNNE- Av. Las Heras 727- Resistencia (Chaco)
}

\begin{abstract}
RESUMEN
Stylosanthes guianensis es una leguminosa forrajera nativa de potencial valor en la producción ganadera del NEA. El objetivo de este trabajo es determinar el efecto de dos alturas de defoliación sobre el rendimiento estacional de materia seca (MS) de esta especie. La siembra se realizó en octubre de 2008, en un suelo Arguidol vértico, con semillas previamente escarificadas, a una densidad de fue $4 \mathrm{~kg} \mathrm{ha}^{-1}$. Se evaluaron dos alturas de defoliación $\mathrm{T}_{1}: 20 \mathrm{~cm} \mathrm{y} \mathrm{T}_{2}: 30 \mathrm{~cm}$. El diseño experimental fue en bloques al azar con cuatro repeticiones, siendo el tamaño de la parcela de $4 \mathrm{~m}^{2}$. En Diciembre se realizó el corte de emparejamiento. Para determinar el rendimiento de MS en cada tratamiento se marcaron puntos fijos donde se cortó una superficie de $0,25 \mathrm{~m}^{2}$. Se determinó el porcentaje de MS y el rendimiento estacional y rendimiento acumulado. Los cortes se realizaron en marzo, octubre y diciembre de 2009 y en marzo de 2010. Se realizó un ANOVA y las diferencias entre medias se probaron con test de Tukey $(\mathrm{p} \leq 0,05)$. El porcentaje de MS para los cortes a $20 \mathrm{~cm}$ varió entre 22,5 y $36 \%$ durante el año, y entre 21 y $31 \%$ para los cortes a $30 \mathrm{~cm}$, correspondiendo los menores valores a la época de activo crecimiento. En el primer corte no se detectaron diferencias significativas entre tratamientos. A partir del segundo corte, las diferencias fueron significativas entre tratamientos. $T_{1}$ tuvo un mayor rendimiento de MS lo que se vio reflejado en una mayor producción acumulada, que fue $1750( \pm 15)$ $\mathrm{g} / \mathrm{m}^{2}$ en $\mathrm{T}_{1}$ y $1236( \pm 43) \mathrm{g} \cdot \mathrm{m}^{2}$ en $\mathrm{T}_{2}$. El corte a $20 \mathrm{~cm}$ permitió obtener un $41 \%$ más de producción. Si bien ambos rendimientos son buenos para especies forrajeras subtropicales, dada la mayor biomasa obtenida, sería recomendable para $S$. guianensis una altura de defoliación de $20 \mathrm{~cm}$.
\end{abstract}

Palabras clave: Stylosanthes guianensis, defoliación, rendimiento de MS

\begin{abstract}
SUMMARY
Stylosanthes guianensis is a native legume with potential forage use in animal production. The aim of this work was to determined the effect of two defoliation height on its seasonal dry matter (DM) yield. Sown was performed on October 2008, on an Arguidol Vertic soil, with scarified seeds, at a rate of $4 \mathrm{~kg} . h \mathrm{~h}-1$. Two cutting height were tested: T1:20 $\mathrm{cm}$ and T2: $30 \mathrm{~cm}$. Experimental design was block at random with four replications. In December a cleaning cut was done. In each treatment fixed points were placed and a sample of $0.25 \mathrm{~m}^{2}$ was cut. Percentage of DM and seasonal and accumulated yield were determined. Cuts were performed in March, October and November 2009 and March of 2010. ANOVA and Tukey test were used to analyzed results. DM percentage at $20 \mathrm{~cm}$ height varied between 22,5 and $36 \%$. For $30 \mathrm{~cm}$ height varied between 21 and 31\%. Minimum values corresponded to active growing season. At first cut differences were not detected among treatments. After that, differences were detected. T1 had higher yield, the accumulated forage was $1750( \pm 15) \mathrm{g} / \mathrm{m} 2$ and 1236 $( \pm 43)$ g.m2 for T2. Cuttings at $20 \mathrm{~cm}$ allowed a $41 \%$ increased yield. So for this species a suitable cutting height would be $20 \mathrm{~cm}$.
\end{abstract}

Key words: Stylosanthes guianensis, defoliation, DM yield

\section{INTRODUCCIÓN}

Stylosanthes guianensis es una leguminosa forrajera nativa de potencial valor para la producción ganadera del NEA (Tomei et al., 1997; Ciotti et al., 1998). Su ciclo de crecimiento es primavero-estivootoñal. S. guianensis se adapta a un amplio rango de condiciones climáticas y edáficas y presenta parámetros de calidad superiores a otras leguminosas tropicales (Machado y Chao, 1980). Es una especie poco exigente en fósforo (P) (Ciotti et al. 2003), por lo cual se adapta a los suelos de la provincia de Corrientes.
En los suelos arenosos se logró su implantación y permanencia en el tapiz vegetal con un adecuado manejo (Ciotti et al., 1999b; Ciotti et al., 2004). En los pastizales del Sur de la Provincia puede intersembrarse con buenos resultados (Royo Pallares y Pizzio, 1988).

Tiene un sistema radicular profundo y extenso, lo que le permite soportar sequías y anegamientos temporarios. Se inocula fácilmente con rizobios presentes en el suelo, aunque se observa que nodula mejor en el segundo año (Ciotti et al., 2006). La producción de forraje varía de 10 a 18 tn ha $^{-1}$ (Ciotti et al., 
1999a) y produce abundantes semillas si se realiza un manejo adecuado del cultivo. Puede ser utilizada en pastoreo directo o como forraje conservado (Ciotti et al., 2009). Es una especie muy sensible al manejo en lo que se refiere a la altura de corte, consecuencia del hábito de crecimiento. Alima (1974) concluyó que la posición relativa de las yemas con respecto al ápice del tallo es un factor importante para el crecimiento. Propuso reemplazar el concepto de altura de corte por el de índice de corte (cutting index) para obtener un rebrote satisfactorio. Funes y Yepes (1974) obtuvieron mayor rendimiento en esta especie forrajera cuando cortaron a $15-20 \mathrm{~cm}$ que cuando lo hicieron a alturas menores $(5-10 \mathrm{~cm})$. En ensayos previos de frecuencia de corte, la altura utilizada fue de 15 a 20 cm (Ciotti et al., 2002).

El objetivo de este trabajo fue determinar el efecto de dos alturas de defoliación sobre el rendimiento estacional de materia seca (MS) de S. guianensis cv. Graham.

\section{MATERIALES Y MÉTODOS}

El ensayo se realizó en la unidad experimental de la cátedra de Forrajicultura de la Facultad de Ciencias Agrarias (UNNE) en un suelo de la serie Puerto Corazón, clasificado como Argiudol vértico (Escobar et al., 1996).

Los parámetros climáticos (temperatura y precipitación) registrados durante el período de octubre 2008 - agosto 2010 y los promedios históricos de 40 años (1950-1990) se detallan en la figura 1.

La especie utilizada fue $S$. guianensis cv. Graham, sembrada a una densidad de $4 \mathrm{~kg} \mathrm{ha}^{-1}$ de semillas, en cuatro surcos de cuatro metros cada uno, distanciados a $70 \mathrm{~cm}$. Las mismas fueron previamente escarificadas con agua caliente a $80{ }^{\circ} \mathrm{C}$ durante 10 minutos. No se realizó la inoculación porque en el sitio ya se habían realizado ensayos con esta especie.

La siembra se realizó el siete de octubre de 2008. Las parcelas fueron regadas de forma manual de acuerdo a las necesidades del cultivo. Se aplicó un diseño en bloques al azar con cuatro repeticiones y las parcelas fueron de $2 \mathrm{~m}^{2}$. Los tratamientos aplicados fueron dos alturas de defoliación $\mathrm{T} 1=$ a $20 \mathrm{~cm}$ y $\mathrm{T} 2=$ a $30 \mathrm{~cm}$ del nivel del suelo.

El control de malezas se realizó en forma manual. Se midió cobertura 20 días luego de la siembra, mediante evaluación visual sobre la superficie de las parcelas. Se evaluó incidencia de plagas y enfermedades, utilizando la escala para evaluación de Ensayos Agronómicos de la Red de Ensayos de Pasturas Tropicales donde $1=$ sin daño y $5=$ daño severo (Toledo, 1982).

En diciembre (17/12/08) se realizó un corte de emparejamiento, a $20 \mathrm{~cm}$ de altura y a partir de allí comenzaron los cortes estacionales de defoliación. Se midió altura y largo de guías de 10 plantas elegidas al azar previo a los cortes. Esta especie inicia su floración después de la primera quincena de marzo, razón por la cual a partir del corte de marzo no se volvieron a cortar las parcelas para asegurar la formación de flores y semillas.

Para determinar el rendimiento de materia seca en cada tratamiento se marcaron puntos fijos donde se cortó una superficie de $0,25 \mathrm{~m}^{2}$. Las muestras fueron pesadas en verde, luego llevadas a estufa hasta peso constante y se determinó el porcentaje de materia seca

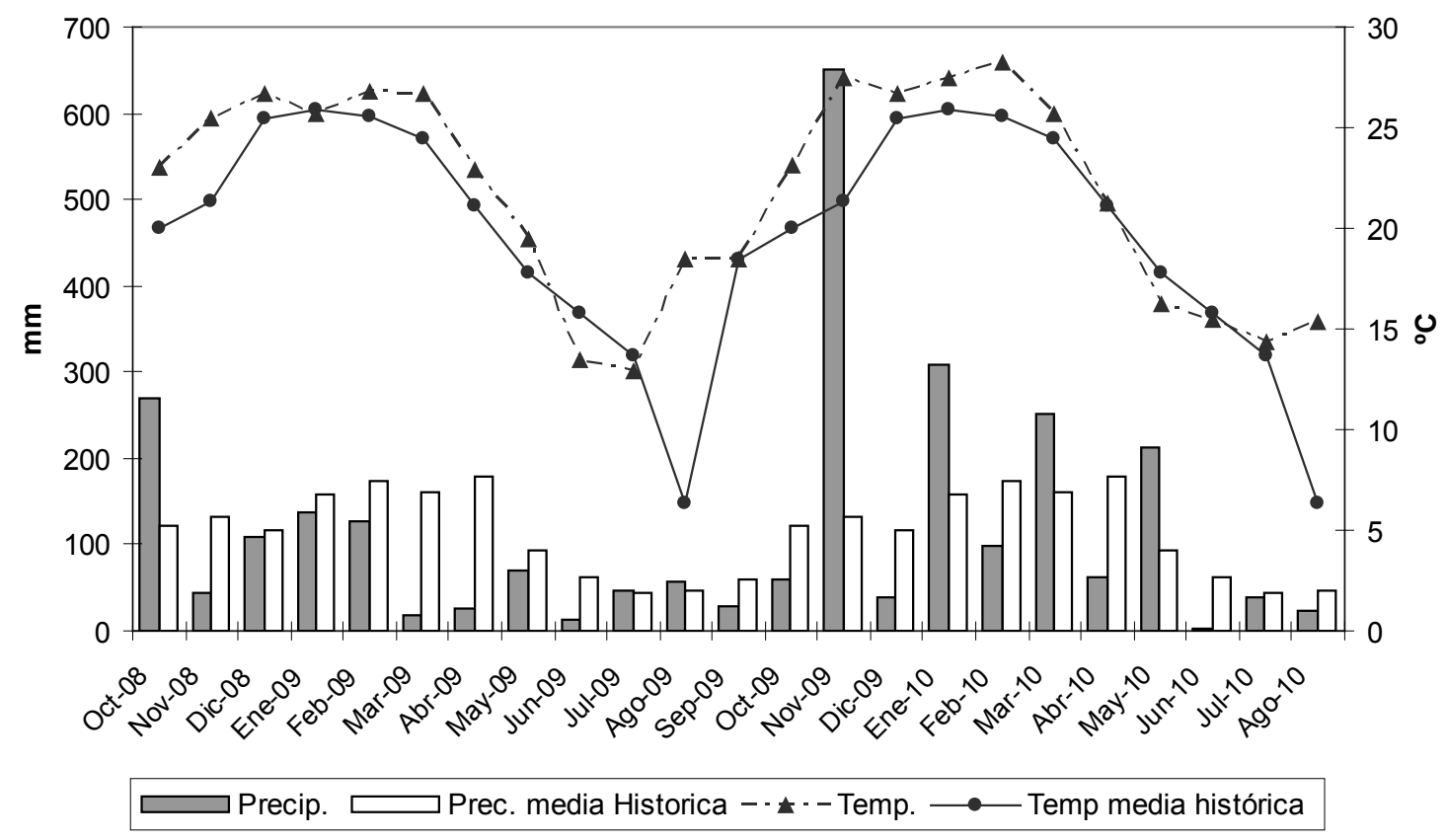

Figura 1: Temperaturas medias mensuales y precipitaciones registradas durante el período de evaluación y promedios históricos (período 1950-1990). Fuente: EEA Colonia Benítez (Chaco). 
Tabla No 1: Rendimientos promedio de MS $\left(\mathrm{kg} \mathrm{ha}^{-1}\right)$ de Stylosanthes guianensis cv. Graham en cada fecha de corte y altura de defoliación.

\begin{tabular}{|c|c|c|c|c|c|}
\hline \multirow{2}{*}{ Tratamiento } & \multicolumn{4}{|c|}{ Fechas de corte } & \multirow{2}{*}{ Total } \\
\hline & $03 / 2009$ & $10 / 2009$ & $12 / 2009$ & $03 / 2010$ & \\
\hline $20 \mathrm{~cm}$ & $892 \mathrm{a}$ & $453,3 \mathrm{~b}$ & $1233,3 \mathrm{~b}$ & 922,7 b & 3388,3 \\
\hline $30 \mathrm{~cm}$ & $800 \mathrm{a}$ & $340 \mathrm{a}$ & $668 \mathrm{a}$ & $664 a$ & 2585,3 \\
\hline DMS & 187 & 109 & 1331,2 & 168,4 & \\
\hline $\mathrm{CV}$ & 9,6 & 11,2 & 6,11 & 9,4 & \\
\hline
\end{tabular}

* Letras distintas en una columna indican diferencias significativas (Tukey $\mathrm{P}<0,05$ )

y el rendimiento, expresado en $\mathrm{kg} \mathrm{ha}^{-1}$. Luego de cada muestreo se realizaron cortes de emparejamiento en todas las parcelas. Los cortes se realizaron en marzo, octubre y diciembre de 2009 y en marzo de 2010.

En julio de 2009 se realizó un corte para cosechar semillas, cortando los primeros $10 \mathrm{~cm}$ de la canopia, en forma manual a toda la parcela.

Los resultados fueron analizados con análisis de varianza y los promedios comparados con el test de Tukey $(\mathrm{p}<0,05)$.

\section{RESULTADOS Y DISCUSIÓN}

El periodo de evaluación de la variable altura de defoliación abarcó desde octubre de 2008 hasta marzo de 2010. Durante el primer año las temperaturas promedio registradas fueron similares al promedio histórico (gráfico 1). Las precipitaciones fueron $50 \%$ inferiores a lo normal (383 $\mathrm{mm}$ vs $759 \mathrm{~mm}$ ).

A los 20 días de la germinación, la cobertura fue mayor al $95 \%$. La altura promedio de las plantas a los 40 días fue $25 \mathrm{~cm}$ y el largo de las guías $69 \mathrm{~cm}$. En un ensayo realizado previamente en un suelo arenoso de la localidad de Santa Ana (Corrientes), la altura registrada a los 30 días fue $40 \mathrm{~cm}$ y el largo de guías $50 \mathrm{~cm}$ (Ciotti et al., 1997). Esta diferencia se debe a que el crecimiento de esta especie es más rápido en suelos de textura arenosa.

La curva de producción de esta especie se extiende desde octubre a junio, alcanza su máximo a fines del verano y principios de otoño (marzo), manteniendo una producción elevada hasta fines de mayo. Con las bajas temperaturas su crecimiento es lento.

\section{Rendimiento de materia seca}

Los rendimientos promedio de materia seca $(\mathrm{kg}$ MS ha ${ }^{-1}$ ) para cada fecha de corte y altura de defoliación se presentan en la tabla $\mathrm{N}^{\circ} 1$.

En el corte de marzo del primer año no se detectaron diferencias significativas entre tratamientos. El rendimiento alcanzado fue superior al rendimiento obtenido en el ensayo realizado en un suelo arenoso de la localidad de Santa Ana, Corrientes (Ciotti et al., 1997). Los valores duplicaron el rendimiento promedio de $480 \mathrm{~g} \mathrm{~m}^{-2}$ de dicho ensayo, cortado a $20 \mathrm{~cm}$ de altura. Esta diferencia debe atribuirse a la diferencia en la densidad de plantas $\left(69 \mathrm{pl} \mathrm{m}^{-2} \mathrm{vs} 53 \mathrm{pl} \mathrm{m}^{-2}\right)$ y al tipo de suelo entre ambos ensayos.

En el corte de octubre se detectaron diferencias significativas entre tratamientos, siendo el rendimiento del corte a $20 \mathrm{~cm}$ un $25 \%$ superior a la otra altura de defoliación.

A partir del mes de octubre se produjo un crecimiento muy activo de guías y hojas, en respuesta a las condiciones climáticas favorables. Esto determinó un incremento en el rendimiento de forraje en ambos tratamientos para el mes de diciembre. Tanto en diciembre como en marzo se mantuvieron las diferencias entre tratamientos, siendo el de $20 \mathrm{~cm}$ significativamente superior al de $30 \mathrm{~cm}$.

Blouard y Turiaux (1962) en ensayos con S. guianensis en el Congo (Africa), determinaron el rendimiento de materia seca con dos frecuencias (tres y cuatro meses) y dos alturas de defoliación (15 y 25 $\mathrm{cm})$. Cuando se cortó cada 3 meses a $15 \mathrm{~cm}$ se obtuvieron rendimientos de $7.281 \mathrm{~kg}$ ha año $0^{-1}$. A $25 \mathrm{~cm}$ los rendimientos fueron $6.785 \mathrm{~kg}$ ha año ${ }^{-1}$. Con una frecuencia de corte de 4 meses, los rendimientos fueron: $6.845 \mathrm{~kg}$ ha año-1 y $6.529 \mathrm{~kg}$ ha año $0^{-1}$ para los cortes a 15 y $25 \mathrm{~cm}$ respectivamente (Skerman et al., 1991).

Grof y Harding (1968) recomendaron utilizar frecuencias de defoliación menores a 18 semanas ya que los tallos inferiores se volvían leñosos y afectaba la persistencia del cultivo. S. guianensis persiste con pastoreos a intervalos de 8 semanas en la República de las Islas Fiji, al sur del Océano Pacífico. Cuando la pastura no es utilizada durante dos años las plantas se vuelven leñosas. Defoliaciones intensas, menores a $20 \mathrm{~cm}$, también resultan en detrimento del cultivo (Skerman et al. 1991).

\section{Porcentaje de materia seca}

El porcentaje de MS para los cortes a $20 \mathrm{~cm}$ varió entre 22,5 y $36 \%$ durante el año, correspondiendo los menores valores a la época de activo crecimiento. El porcentaje de MS para los cortes a $30 \mathrm{~cm}$ varió entre 21 y $31 \%$ a lo largo del año. Estos valores son similares a los obtenidos en ensayos anteriores con ésta especie (Ciotti et al., 1997). 


\section{Rendimiento de semillas}

Las plantas de $S$. guianensis iniciaron la floración el 10 de mayo de 2009 y alcanzaron la plena floración el 13 de junio de 2009.

Se consideró inicio de maduración de frutos el momento en que, al golpear las inflorescencias contra la palma de la mano, caían 1 o 2 semillas en el $5 \%$ de las inflorescencias (González y Mendoza, 1994). La cosecha se realizó entre los 14 y 21 días posteriores al inicio de maduración, de acuerdo al momento de cosecha determinado para esta especie (Ciotti et al., 2000).

El rendimiento promedio fue $14,5 \mathrm{~g} \mathrm{~m}^{-2}$, lo que equivaldría a $145 \mathrm{~kg}$ de semilla ha ${ }^{-1}$. Esta producción de semillas fue la esperada para las condiciones del Norte de Corrientes (Ciotti et al. 1999a)

\section{CONCLUSIONES}

Los rendimientos de materia seca obtenidos con cortes a 20 y $30 \mathrm{~cm}$ pueden ser considerados buenos para especies forrajeras subtropicales. El corte a 20 $\mathrm{cm}$ permitió obtener un $41 \%$ más de producción. Por este motivo, sería recomendable para $S$. guianensis una altura de defoliación de $20 \mathrm{~cm}$.

Por el rendimiento de semillas obtenido podría considerarse una actividad complementaria a su uso como forraje.

\section{BIBLIOGRAFIA}

Alima G, B. 1974. Preliminary studies on the biological effects of cutting height on the growth an development of Stylosanthes gracilis H.B.K: Cohiers ORSTOM, Biologie No 23:57-65.

Blouard,R. y Turiaux, L 1962. Stylosanthes gracilis, son comportement et son utilisation au Congo. Bull. Inf. INEAC, 11(4-6):339-355.

Ciotti, E. M; Tomei, C. E; Castelan, M. E.1997.Curva de producción y frecuencia de corte de Stylosanthes guianensis CIAT 184. Revista Argentina de Producción Animal. Vol. 17 Suplemento 1: 148-150.

Ciotti, E.M;Castelan, M. E.; Tomei, C.E. 1999a. Perspectivas de la producción de semillas de Stylosanthes guianensis en Corrientes. Agrotecnia $\mathrm{N}^{\circ} 5: 21-25$.

Ciotti, E.M; Tomei, C. E; Castelan, M. E. 1999b. The adaptation and production of some Stylosanthes species in Corrientes northwest. Tropical Grasslands 28:165-169.

Ciotti, E. M., Castelan, M. E; Tomei, C. E. 2000. Momento de cosecha de Stylosanthes guianensis CIAT 184, Agrotecnia $\mathrm{N}^{\circ}$ 6:14-17.
Ciotti, E M., Castelán, M. E., Tomei, C. E. 2002 Stylosanthes guianensis: densidad de siembra y frecuencia de cortes. Rev. Arg.de Prod. An., Vol: 22 (3-4): $185-190$

Ciotti, E. M.; Castelan, M. E.; Tomei, C. E., Mónaco, I. P; Benitez, J. A. 2003. Respuesta de Stylosanthes guianensis CIAT 184 a la fertilización con una baja dosis de fósforo. Revista de Investigaciones Agropecuarias (RIA), 32 (2): 137-145.

Ciotti, E.M., Castelán, M.E., Tomei, C.E., Acuña, D. 2004. Intersiembra de Stylosanthes guanensis en un pastizal del NO de Corrientes. Reunión de Comunicaciones Científicas y Técnicas UNNE: 4 pp

Ciotti, E. M., Castelán, M. E., Berg, C. H. 2006. Efecto del encharcamiento temporario sobre el rendimiento y la nodulación de Stylosanthes guianensis y Arachis pintoi. Pasturas Tropicales Vol. 28 (1): 52- 56

Escobar, E.;,Ligier, H.; Melgar, R.; Matteio, H; Vallejos,O. 1996. Mapa de suelos de la Provincia de Corrientes 1:500.000. INTA, EEA Corrientes, $329 \mathrm{pp}$.

Funes, F. y Yepes, S. 1974 Pasture introduction in Cuba. Proc. Of $12^{\text {th }}$ Inst. Grassld. Congr.Plant. Int. Breed and seed Prod Moscow. URSS. 89-104.

González, Y; Mendoza, F. 1994. Momento de cosecha de Stylosanthes guianensis. Rev. Pastos y Forrajes, Vol 17: 225.

Grof;B y Harding; W. 1968. Stylosanthes guyanensis frequency of cutting trial. Queensl.Dept.Prim. Ind. Agrost. Tech. Ann. Rep.1: 14-15.

Royo Pallares, O. y Pizzio, R. 1988. Introducción de especies para el mejoramiento del campo natural en el Sur de Corrientes, Argentina. XIV Reunión Grupo Campos, INIA Tacuarembó: 31-38.

Skerman, P.J; Cameron, D.G.; Riveros, F. 1991. Leguminosas forrajeras tropicales. Colección FAO $\mathrm{N}^{\circ} 2$ : Producción y protección vegetal. Roma: 425-438.

Tepper, N. E; Ciotti, E. M.; Porta, M. 2009. Producción estival de materia seca de Stylosanthes guianensis con dos alturas de corte. XX Reunión de Comunicaciones científicas, técnicas y de extensión, Facultad de Ciencias Agrarias UNNE, Corrientes.

Toledo, J.M. 1982 Manual para la evaluación agronómica, Red internacional de evaluación de pastos tropicales, CIAT: 45-72.

Tomei , C.E.; Ciotti, E.M,; Castelan, M.E. 1997. Stylosanthes, alfalfa tropical. Boletín $\mathrm{N}^{\circ} 7$, Inst. Agrot. Fuentes Godo: 7 pp. 